\title{
The impact of collarette region-based convolutional neural network for iris recognition
}

Original Scientific Papers

\author{
Souheila Tounsi \\ Higher School of Industrial Technologies (ESTI), \\ LERICA Laboratory, Badji Mokhtar-Annaba University, Annaba, Algeria \\ Annaba city, P.O Box 218 23000, Annaba, Algeria. \\ tounsi@esti-annaba.dz
}

\section{Karima Boukari}

Badji-Mokhtar University, Faculty of Technology, Department of electronics, LERICA Laboratory, Badji Mokhtar-Annaba University, Annaba, Algeria Annaba University, P.O. Box 12, 23000 Annaba, Algeria boukkarima@yahoo. Fr

\author{
Abdourazek Souahi \\ Badji-Mokhtar University, \\ Faculty of Sciences, Department of mathematics, \\ Laboratory of advanced materials, Badji Mokhtar-Annaba University, \\ Annaba, Algeria \\ Annaba University, P.O. Box 12, 23000 Annaba, Algeria \\ arsouahi@yahoo.fr
}

\begin{abstract}
Iris recognition is a biometric technique that reliably and quickly recognizes a person by their iris based on unique biological characteristics. Iris has an exceptional structure and it provides very rich feature spaces as freckles, stripes, coronas, zigzag collarette area, etc. It has many features where its growing interest in biometric recognition lies. This paper proposes an improved iris recognition method for person identification based on Convolutional Neural Networks (CNN) with an improved recognition rate based on a contribution on zigzag collarette area - the area surrounding the pupil - recognition. Our work is in the field of biometrics especially iris recognition; the iris recognition rate using the full circle of the zigzag collarette was compared with the detection rate using the lower semicircle of the zigzag collarette. The classification of the collarette is based on the Alex-Net model to learn this feature, the use of the couple (collarette/CNN) allows for noiseless and more targeted characterization and also an automatic extraction of the lower semicircle of the collarette region, finally, the SVM training model is used for classification using grayscale eye image data taken from (CASIA-iris-V4) database. The experimental results show that our contribution proves to be the best accurate, because the CNN can effectively extract the image features with higher classification accuracy and because our new method, which uses the lower semicircle of the collarette region, achieved the highest recognition accuracy compared with the old methods that use the full circle of collarette region.
\end{abstract}

Keywords: Iris Recognition, Collarette zigzag, CNN, CASIA-Iris-Lamp V4, biometric, SVM.

\section{INTRODUCTION}

Making an application more secure and less accessible to unwanted people requires discerning one person from another. There are several ways to identify a person and biometrics is one of the most secure alternatives so far.

An iris is a spherical membrane of the eye, among the sclera and pupil. It starts to shape throughout the embryo phase, completing its formation at about eight months of age. The originality of the iris lies in the richness of texture details resulting from the radial furrows, crypts, filaments, flecks, pigment frills, stripes, arching ligaments, and collarette. This richness makes the human iris one of the most reliable biometric characteristics [1]. The area of the iris is composed of two regions, the outer ciliary zone, and the central pupillary zone. The area between these two regions is the collarette. The collarette contains sufficient discriminating characteristics. It is less affected by pupil dilation and is 
generally unaffected by eyelashes and eyelids [2]. Fig. 1 shows a human iris.

This paper proposes an improved iris recognition method for person identification based on CNN and zigzag collarette. The iris detection rate using the full circle of the collarette region was compared with the lower semicircle of the collarette area. Our approach of using the latter one is evaluated on images from the CASIA-Iris-Lamp V4 database. This version 4 of the CASIA database has been applied for the first time to test the effect of the zigzag collarette region using convolution networks (CNNs) as feature extractors.

The rest of this paper is as follows: Section 2 gives a short review of past works that used iris recognition and collarette area, Section 3 explains our proposed approach including experimental results and analysis and Section 4 provides the conclusion of our work.

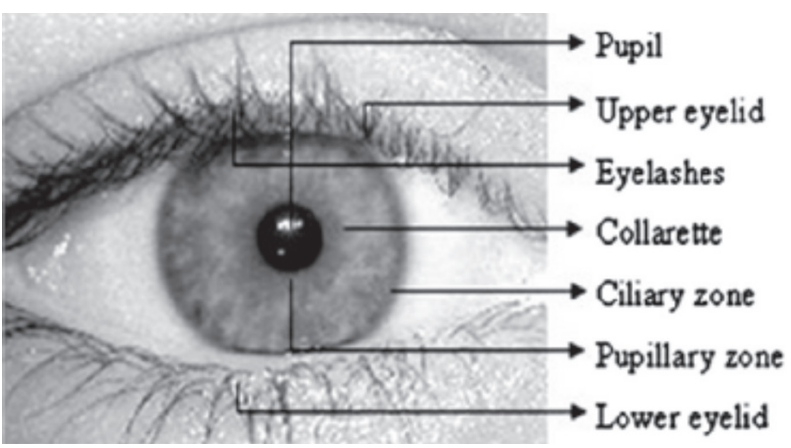

Fig. 1. Human eye

\section{RELATED WORK}

In recent years, the field of biometric identification has undergone remarkable development. Because of its high reliability and its importance in many fields.

- In the papers [3], the author proposes a highlevel security algorithm for the encryption of iris images by combining the permutation method, the QR code, and the chaotic system.

- In the papers [4] an algorithm is proposed to detect a type of intraocular cancer called Uveal Melanoma (UM), the proposed method uses iris segmentation algorithms and proposes a new algorithm for the detection of UM using fuzzy logic and artificial neural networks.

- Iris recognition is used in [5] in combination with fingerprints and palmprints for multimodal biometric verification at the final decision stage. In [6], the authors segmented the iris as follows: the detection of eye regions was done via an unsupervised neural network, the eye contour was determined using the canny filter, and the pupil and iris using the Hough.

- $\quad$ In [7], the authors used a graphical user interface for the segmentation of the iris image; this interface uses active contours for non-cooperative biometric recognition to localize the iris structure.

- A new proposal for detecting eyelashes and eyelids with the least noise is discussed in [8], this proposal reduces the detection time of eyelashes and eyelids in the iris image using the Hough transform.

- $\quad$ In [9], the authors presented a new scheme for cancelable iris recognition system based on comb filtering, the author used in his system a coarse to fine the iris localization, then feature extraction using Gabor filtering.

- In the paper [10], the proposed idea adopts the first two phases of Daugman's approach, localization, and standardization. After the normalization stage, the system uses the Base64 coder to convert the normalized image of the iris, into plain text, then extract the language-independent features of the resulting text without modifying its statistical properties, which leads to a numerical model, the resulting model will be later classified using machine learning algorithms such as Random Forest.

- In paper [11], the authors used the circular Hough transform for segmentation to find the region of interest $(\mathrm{ROI})$ of images of the eye, after that Daugman's Rubber sheet model is used for normalization. Then, for feature extraction, the GLCM technique is used. Finally, Discriminant analysis is applied for the classification of the images.

- In [12], the authors proposed a new method of classification and feature extraction based on the hybrid classifier MLPNN-ICA and grey level difference and the hybrid classifier MLPNN-ICA.

- In [13], the authors designed an iris recognition system consisting of segmentation where the author implemented the Canny edge detection algorithm for edge detection, rubber sheet model for normalization, Gabor filter for feature extraction using and hamming distance for classification.

- In [14], a secure vault system based on an iris recognition system is proposed, this system uses a point-to-point feature pattern-matching algorithm and $\mathrm{PIC}$ microcontroller.

In the previous section of the paper, recent work on iris detection has been presented to show what has been done recently in the field of iris detection.

In the previously mentioned works, the authors focused on the iris region. Despite the effectiveness of the above-mentioned methods for less noisy images, they often suffer a serious drop in performance when confronted with very irregular or poorly segmented masks. A solution to this problem is therefore needed 
for robust, complete, and more accurate iris segmentation.

Motivated by the previous works, this paper is focused on the area of the collarette zigzag, and more precisely the lower semicircle of the collarette zigzag (see Fig. 5). The choice of the area to be studied - the lower semicircle of collarette zigzag - was not arbitrary as it is an area of the iris with a rich texture, unaffected neither by the eyelids nor by the eyelashes. The main contribution of our idea is to select the most important region of the iris, a more complex pattern, with less noise, to avoid another noise removal treatment that reduces the image quality in most cases.

In fact, the zigzag collarette region is one of the most important parts of the iris pattern because of its rich texture, its insensitivity to pupil dilation, and because it is not affected by the eyelash or eyelid. In [15], the authors found empirically that the zigzag collarette is generally concentric with the pupil and that its radius is restricted within a certain interval.

The use of the collarette area in iris recognition has led to many advances over the last decade:

- In [16], collarette boundary detection is used to improve the recognition rate. Histogram equalization and a high-pass filter are applied, after using a one-dimensional DFT, the authors used statistical information from the image to detect the collarette boundary.

- In [15], the method is based on the zigzag collarette area and crossed chord Theorem.

- In [17], based on the zigzag collarette area localization and an asymmetrical support vector machine, an effective iris recognition technique is presented.

- In [18], the authors presented the experiments by using different normalization algorithms and different iris radii in iris recognition steps. They proposed an iris localization method and a collarette localization method. In feature extraction, they proposed the Gabor wavelet filter to extract characteristics from iris images.

- In [19], the author used the Haar wavelet to localize the zigzag collarette and used a 1D Log Gabor filter for feature extraction.

- In [20], a combination of Support vector machine (SVM), artificial neural networks (ANN), and Zigzag collarette area are used to perform feature extraction for iris recognition system.

- In [2], the author proposes a new feature extraction technique using wavelets [21] combined with DLDA [22] to extract discriminative low-dimensional feature vectors from the collarette region.

- In [23] iris segmentation and normalization, algorithms based on zigzag collarette are presented.
The authors used canny edge detection and Hough transforms to localize pupil near the zigzag area. After that, the use of a Daugman Rubber Sheet Model represents an isolation zigzag collarette.

- In [24], the authors used the chain code method and zigzag collarette area with a Support Vector Machine (SVM) to enhance the iris recognition method for person identification.

- In [25], the author used a two-level segmentation method to segment the image. In the inner boundary segmentation section, They used methods such as Gauss pyramid, anisotropic scattering, thresholding, etc. . In the outer boundary, segmentation section the authors performed a zigzag collarette process using zigzag collarette methodology. Finally, the inner boundary segmentation was subtracted from the outer boundary segmentation to give the segmented iris.

Iris's recognition process consists of several steps. First, segmenting the iris region is done. After that, the normalization is carried out to transform images from Cartesian to polar coordinates. Then, the features extraction step, which is necessary to detect the features in the last stage of the classification, see [26].

Extracting efficient characteristics is the major important stage in many object recognition tasks. That is why many researchers have focused on proposing robust features for a variety of image classification stages, see [27] and references cited therein. Nowadays, a lot of attention is given to Convolutional Neural Networks (CCN) and feature learning algorithms. In this algorithm, the image is transmitted directly to the CNN, and then the algorithm extracts the best features image, see $[17,27,28]$.

In addition to feature extraction, the researchers have attempted, through the use of the $C N N$, to eliminate the drawbacks of all current segmentation methods and replace much of the pre-processing and post-processing. All these advantages justify our choice to use the CNN method. Learning-based methods are an advanced type of segmentation method, as stated in [29]. Among all learning-based methods, deep learning using deep CNN is among the best known and best learning popular methods in current computer vision applications because of its accuracy and performance. Deep CNN has been implemented to detect damaged road marks [30], recognize human gender from human body images [31], detect people in night environments using a visible light camera [32] and it used for spatial feature extraction to classify lung ultrasound (LUS) videos for diagnosing COVID-19 [33]. As with CNN's brain tumor segmentation, CNN can also provide a solid platform to facilitate intensive work with accuracy and efficiency, see [34,35].

Iris applications are sensitive because they have a very complex texture. Therefore, to the best of our 
knowledge, there are not many research papers on CNN related to iris segmentation, the following are the most known ones.

- $\quad$ In [36], CNNs are used for verification purposes and to learn relational characteristics. Also, to calculate the similarity between two iris candidates the authors used Deeplris on heterogeneous iris images.

- In [37], the authors used Deeplris Net for two research studies that focus on iris recognition rather than iris segmentation. These two studies are a visual representation of the iris and iris detection by crossed sensors.

- In [35], using fully convolutional networks (FCNs), the authors detected precise iris boundaries in non-cooperative environments. In his work, hierarchical CNNs (HCNNs) and multi-scale FCNs (MFCNs) have been used to automatically delineate iris boundaries.

- In [38], the authors used CNN entropy-based clustering to effectively segment the iris, sclera, and pupil regions. Here, CNN does the segmentation using entropy measurements.

- In [39], the authors proposed iris segmentation models based on deep learning to highlight very irregular texture areas in post-mortem iris images. The article proposed a very efficient approach to iris segmentation, called IrisParseNet based on deep learning (CNN), which differs from many iris segmentation methods.

- This article makes an interesting study by explaining the limitations of the traditional approach and the advantages of the deep learning approach for iris recognition [40].

Recent works [17,26,27,35-42] are focused on iris recognition with the $C N N$, but in our case, we have replaced the couple iris/CNN by the couple collarette/ CNN in order to take advantage of both: CNN is the best known and most popular deep learning in current computer vision applications because of its accuracy and performance as it can efficiently extract features from the image with higher classification. In addition, deep neural networks learn high level features in hidden layers; this is one of the biggest strengths of CNN. It reduces the need for feature engineering. CNNs also correct the drawbacks of all current segmentation methods as a replacement of a large part of pre-processing and post-processing. All these advantages justify our choice to use the CNN method.

As for the choice of the zigzag collarette, this area is a part of the iris that contains enough discriminating features due to its rich texture and more complex pattern. It is less affected by pupil dilation and is usually not affected by eyelashes and eyelids which makes it less noisy thus avoiding another noise removal processing that reduces the image quality in most cases.

\section{PROPOSED METHOD}

The proposed method involves the implementation of the steps illustrated in Fig. 2. The first step after the acquisition of the image is the pre-processing which consists in eliminating the white points of the iris as well as other types of noise such as eyelashes and noises of salt and pepper in the image of the eye, etc. Thereafter, we pass to the stage of localization of the iris, our goal in this article is not to detect the iris itself but is to detect a part of the iris that is the collarette zigzag (more precisely the lower semi circle), the latter is the subject of our contribution, The detection of the zigzag collarette is done using the Canny contour detector followed by the circular Hough transform, after that, we perform normalization to have a fixed pattern in polar coordinates using the Daugman rubber sheet model, then we move to the stage of feature extraction using the CNN. Our choice was made on the Alex-Net pre-trained model because it is simple and efficient, at the end, a classification step by SVM is necessary to calculate the recognition rate using the accuracy formula.

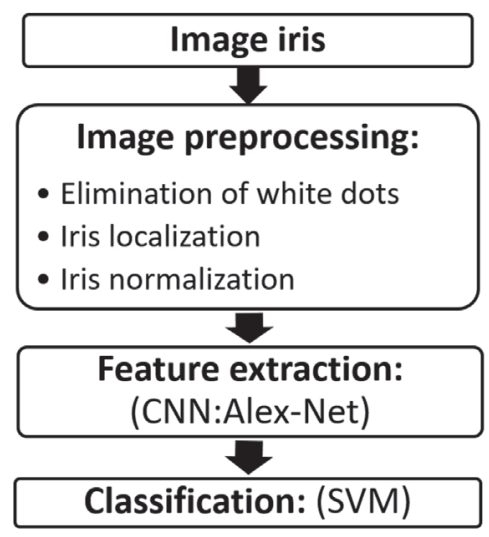

Fig. 2. Stages of iris recognition

\section{1. IMAGE ACQUISITION}

The proposed method was tested on the CASIA-IrisLamp database. One of six subsets was collected using a hand-held iris sensor produced by OKI. A lamp was turned on/off close to the subject to introduce more intra-class variations when CASIA-Iris-Lamp was collected. Different lighting conditions result in the expansion and contraction of the pupil, which causes elastic deformation of the iris texture. This is one of the most common and difficult problems to solve in iris recognition. Therefore, CASIA-Iris-Lamp is good for studying problems of non-linear iris normalization and robust iris feature representation. The CASIA database images are JPG images with a resolution of $640 * 480$. All iris images are 8 -bit grey-level images and the file format is JPEG $(584,2020)$.

\section{2. IMAGE PRE-PROCESSING}

The framework of our recognition system is shown in Fig. 2. In the image pre-processing part, there are three processes which are the elimination of white dots, iris localization, and Iris normalization. 


\section{2. 1. Elimination of white dots}

In most standard iris databases [43-46], white dots may exist in the input eye image and may remain and disrupt the iris localization process if not properly removed, see [47-51]. However, in [52], this paper proposed an effective system to suppress white dots and other extraneous noise such as eyelash threads or salt-and-pepper noise in the eye image, the result is shown in Fig. 3.

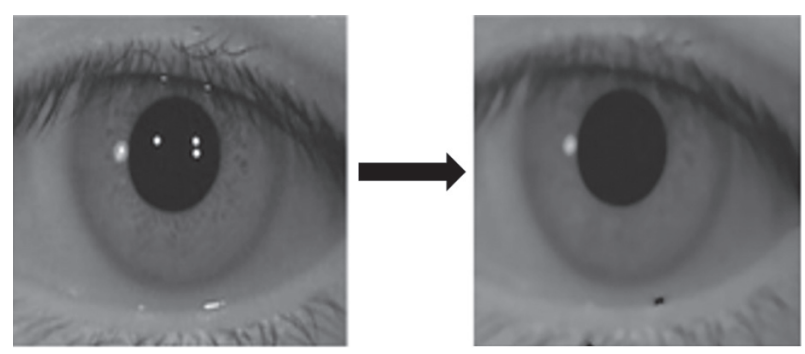

Fig. 3. Elimination of white dots

\section{2. 2. Iris localization (collarette zigzag)}

\section{- Pupil detection}

During the acquisition process, changes in lighting conditions can influence the quality of the resulting iris region, and then affect the localization of the iris and subsequently the recognition result. To improve the accuracy and reliability of an iris recognition system, one must have an accurate localization of the iris region, because the performance of the subsequent steps of the system is directly dependent on the quality of the detected iris region. An ordinary iris localization system aims at detecting the two iris region boundaries: the inner (pupil-iris) boundary and the outer (iris-sclera) boundary. However, the task becomes more difficult, when eyelids and eyelashes cover parts of the iris. For this reason, a new idea has been proposed based on the detection of the collarette- and more precisely the lower semicircle of collarette-, after detecting the pupil, instead of detecting the entire iris.

The iris segmentation process starts with the detection of the pupil boundaries. for this, the Canny Edge detection is applied [53] to generate an edge map, then the circular Hough transform (CHT) is applied [54]. The standard circular Hough transform is used to detect circular shapes from a given radius in the image. The edge detection of the image is based on the calculation of the first derivatives of the intensity values. Each point in the edge map gives a circle of radius $r$ and center (xc, yc) to an output array called accumulators. Then, the largest peak will be searched in the resulting array of accumulators in the parameter space using a voting procedure, the largest peak in the array of accumulators corresponds to the circle best defined by the edge points, as stated in $[55,56]$.

In our experiment, the limit radius of pupils is between 20-60 pixels for the CASIA database. These values were found according to heuristic techniques after examining all the images in the CASIA V4 database. After that, a voting procedure is applied to select the largest peak in the resulting accumulator array, which represents the best drawn circle by the edge points [41]. Finally, the voting procedure is implemented in the Hough space to detect the correct circle as shown in Fig. 4.

\section{- Collarette zigzag region detection}

The next processing is to isolate the zigzag collarette area (see Fig. 5). This area is generally concentric and close to the pupil. Therefore it will be very easy to detect using the formula of tracing a circle using the center coordinates of the pupil and a radius that is equal to 24 according to a study conducted by Rai. It concluded that the zigzag collarette is within 24 pixels of the pupil [19]. In the research of zigzag collarette area detection, many problems are encountered. In most cases, there is noise around the pupil which results in a geometric shape of the pupil that is not completely circular. There are still eyelashes and eyelids covering the area. Hence, researchers in this field tried to make some noise processing, as in paper [42].

In our work, this great problem is overcome by using only the lower semicircle of the collarette zigzag (see Fig. 5) which is neither affected by eyelids nor eyelashes. The principle of our idea is to select the most important region and the most complex pattern with less noise, to avoid another noise treatment that reduces the image quality in most cases.

\section{2. 3. Iris normalization (collarette zigzag)}

Once the zigzag collarette is detected, normalization is implemented to produce a standard size feature vector that allows comparison between two different iris images. Stretching of the iris region is caused by dilation of the pupil with several lighting conditions, changes in the image acquisition distance, rotation of the camera or the eye, elastic distortion of the iris texture. All the above, problems affect the result of the iris comparison and may cause dimensional inconsistencies that should be resolved by normalization. As shown in Fig. 6, the iris normalization process is applied using Daugman rubber sheet mapping to transform the image iris from Cartesian to polar coordinates. The result of normalization is shown in Fig. 7 [42].

Contrast limited adaptive histogram equalization (CLAHE) is a method of contrast adjustment to get an image with uniformly distributed intensity levels. In this paper, CLAHE is applied to the normalized images (see Fig. 7). This latter is enhanced to avoid losing features, extract key points accurately, and hence increase the recognition accuracy.

The new idea here is to take only the lower semicircle of the collarette zigzag (as described previously), and divide the normalized iris into two parts, see Fig. 8. According to the image database, it's very noticeable that 
the lower part of the iris is the least affected by the eyelashes and the eyelids compared to the upper part and in most cases, this part represents a region with zero noise. In the rest of the paper, we compare the recognition rate of the iris using the full circle of collarette and the lower semicircle of collarette to further show the great improvement in the recognition rate.

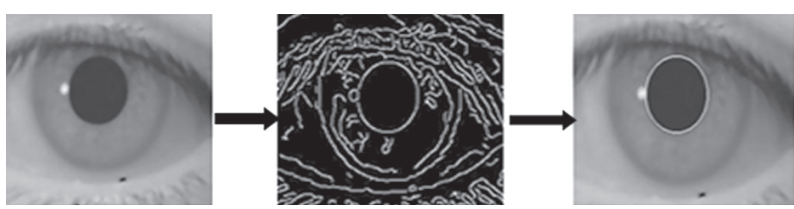

Fig. 4. Pupil detection
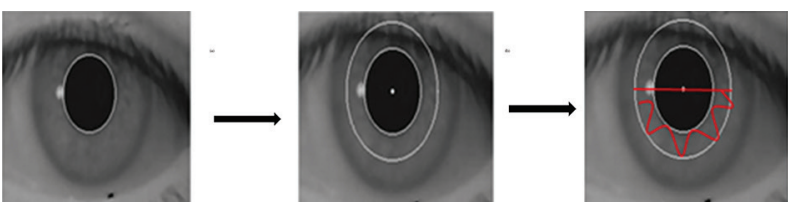

Fig. 5. Isolation of collarette zigzag area (a) and lower semicircle of zigzag collarette area (b).

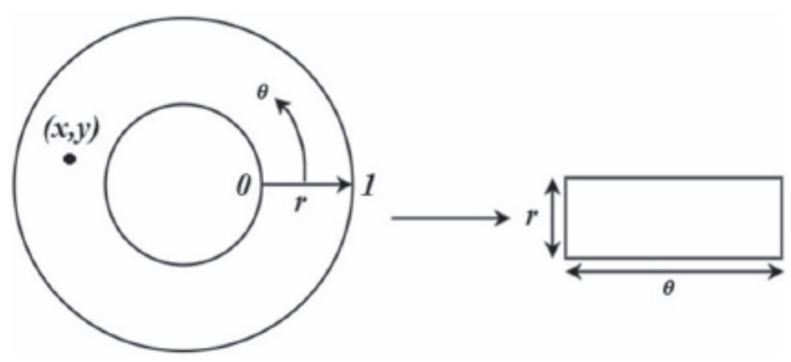

Fig. 6. Transforming the iris region from the Cartesian coordinates to the polar coordinates

\section{3. FEATURE EXTRACTION}

\section{- Convolutional Neural Network (CNN)}

Alex-Net is the pre-trained network used in this paper for the feature extraction process, the choice of the Alex-Net model over another pre-trained model is not arbitrary, Alex-Net is a simple model and it offers the possibility to test performance without compromising memory and time.

The pre-trained (Alex-Net) is a Convolutional Neural Network model CNN a reduced version of the conventional Le-Net [42]. This model was conceived by the Super Vision group [57]. Fig. 9 shows the architecture of Alex-Net and Table 1 explains it further in detail.

Deep neural networks learn high-level features in the hidden layers. This is one of the greatest strengths of $\mathrm{CNN}$ and leads to reduced feature engineering needs. In fact, the image takes several transformations. Firstly, the image goes through many convolutional layers where the network learns new and increasingly complex features. Then, the information from the transformed image passes through the fully connected layers and is transformed into a classification. the highlevel features can be recovered from the last convolutional layers FC7.

To avoid a very long learning time using $C N N$, and since all layers are responsible for learning certain characteristics from the images, features can be retrieved from the network at any time during the training process. We use these extracted features as input data for a classification model with Support Vector Machines (SVM).

In our work, the characteristic vector is recovered from the fully connected layer (FC7) because we get high-level features from these convolutional layers.

\section{4. The classification}

The classifier is applied after feature extraction to find the corresponding label for every test image. A lot of classifiers can be used for classification, with different types. For example, we state the Neural Network, Softmax Regression, and Support Vector Machine, see [56] for more details. In our work, a multiclass Support Vector Machine classifier is used. The SVM is a supervised learning algorithm; it constructs an optimal hyper-plane as a decision surface to maximize the margin of separation between the two classes of data. Support vectors refer to a small subset of the training observations used as support for the optimal location of the decision surface.

After loading the database, it is devised in two parts: $70 \%$ for training and $30 \%$ for testing. Then, we extract the class labels from the training and the test data. The principle of the SVM classification algorithm is to place each data point in an $n$-dimensional space, where $n$ is the number of characteristics. The value of each character is the value of a particular coordinate. Later, we perform the classification by searching for the hyper-plane that differentiates the two classes very well. The SVM algorithm will therefore classify the images and finally calculate the "Accuracy" or learning rate, i.e. the efficiency of the method or the accuracy of the classification. This value expresses the fraction of labels that the network correctly predicts, as defined by the formula (1).

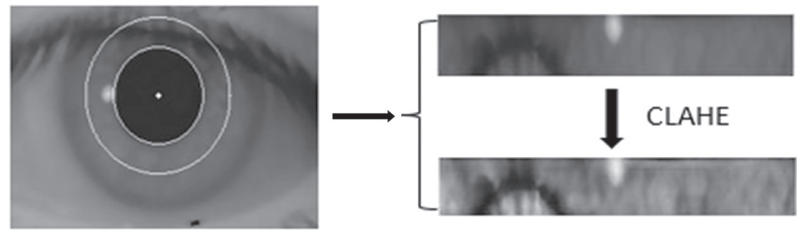

Fig. 7. Iris normalization and enhancement

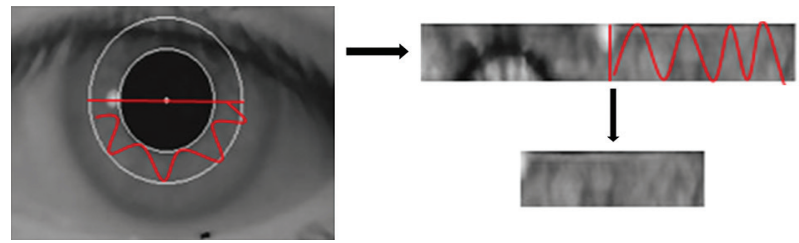

Fig. 8. Iris normalization: lower semicircle of collarette region 
Table 1. The Alex-Net Layer [17]

\begin{tabular}{|c|c|c|c|c|c|}
\hline Type of Layer & 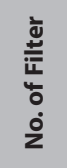 & 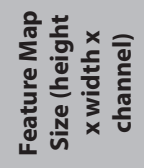 & $\frac{\stackrel{N}{n}}{\frac{ \pm}{2}}$ & $\begin{array}{l}\frac{0}{0} \\
\frac{2}{2} \\
\text { ஸे } \\
\vdots \\
\vdots \\
z\end{array}$ & 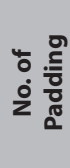 \\
\hline Image input layer & & $227 \times 227 \times 3$ & & & \\
\hline $\begin{array}{l}\text { (1st convolutional } \\
\text { layer) Relu-1 }\end{array}$ & 96 & $55 \times 55 \times 96$ & $11 \times 11$ & $4 \times 4$ & $0 \times 0$ \\
\hline $\begin{array}{l}\text { Cross-channel } \\
\text { normalization } \\
\text { Max pooling } 1\end{array}$ & 1 & $27 \times 27 \times 96$ & $3 \times 3$ & $2 \times 2$ & $0 \times 0$ \\
\hline $\begin{array}{l}\text { (2nd convolutional } \\
\text { layer) Relu-2 }\end{array}$ & 256 & $27 \times 27 \times 256$ & $5 \times 5$ & $1 \times 1$ & $2 \times 2$ \\
\hline $\begin{array}{l}\text { Cross-channel } \\
\text { normalization } \\
\text { Max pooling } 2\end{array}$ & 1 & $13 \times 13 \times 256$ & $3 \times 3$ & $2 \times 2$ & $0 \times 0$ \\
\hline $\begin{array}{l}\text { 3rd convolutional } \\
\text { layer) Relu-3 }\end{array}$ & 384 & $13 \times 13 \times 384$ & $3 \times 3$ & $1 \times 1$ & $1 \times 1$ \\
\hline $\begin{array}{l}\text { (4th convolutional } \\
\text { layer) Relu-4 }\end{array}$ & 384 & $13 \times 13 \times 384$ & $3 \times 3$ & $1 \times 1$ & $1 \times 1$ \\
\hline $\begin{array}{l}\text { (5th convolutional } \\
\text { layer) Relu-5 }\end{array}$ & 256 & $13 \times 13 \times 256$ & $3 \times 3$ & $1 \times 1$ & $1 \times 1$ \\
\hline Max pooling 5 & 1 & $6 \times 6 \times 256$ & $3 \times 3$ & $2 \times 2$ & $0 \times 0$ \\
\hline $\begin{array}{l}\text { Fully connected layer- } \\
\qquad 6(\mathrm{fc} 6)\end{array}$ & & $4096 \times 1$ & & & \\
\hline Relu-6 & & $4096 \times 1$ & & & \\
\hline $\begin{array}{l}\text { Fully connected } \\
\text { layer-7 (fc7) }\end{array}$ & & $4096 \times 1$ & & & \\
\hline Relu-7 & & $4096 \times 1$ & & & \\
\hline $\begin{array}{c}\text { Fully connected } \\
\text { layer-8 (fc8) Softmax } \\
\text { layer }\end{array}$ & & $1000 \times 1$ & & & \\
\hline Output layer & & 1000 class & & & \\
\hline
\end{tabular}

$$
\text { Accuracy }=\frac{\text { correctly } \text { classified Iris Images }}{\text { Total Number }} \times 100
$$

\section{5. EXPERIMENTAL RESULTS AND ANALYSIS}

Our experiments are performed in MATLAB R2018a on a PC Intel core i5, RAM $6 \mathrm{~Gb}$, and a Windows operating system 64 bits. The images used in our experiments come from the CASIA V4-Iris-Lamp image database. This most recent version of CASIA was used with CNN to study the effectiveness of using zigzag collarette region in iris recognition.

For our experiment, 400 images were used from the CASIA database ( 40 classes and 10 samples per class), several experiments are performed with 100 images (10 classes, 10 samples), 200 images (20 classes, 10 samples), $300 \mathrm{im}$ ages (10 classes, 10 samples), 400 images (40 classes, 10 samples) as mentioned in Table 2. Alex-Net is used for feature extraction. The data is divided into two phases, $70 \%$ for training and $30 \%$ for testing. All images in the database are resized to 227 by 227 which is the input size of the Alex-Net. All grayscale images are converted to RGB. At the end, the features learned using the CNN (Alex-Net) and extracted from the layer FC7 will be injected into the multiclass SVM classifier for image classification.
The proposed idea in the previous section has been implemented and the accuracy was calculated using the full circle of the collarette zigzag and compared with accuracy calculated using the lower semicircle of the collarette zigzag. The experimental result is shown in Table 2.

The pre-trained CNN model used was already trained on more than a million images as the feature extractor and the SVM as the classifier. Alex-Net with the SVM classifier achieved good accuracy with a fairly short training time, on the order of seconds to minutes only. The advantage of the pre-trained CNN model is the elimination of the laborious task of feature engineering, making it easier to learn the new assigned task. Table 2 illustrates the accuracy rate with the different cases studied: 100 images, 200 images, 300 images, and 400 images as explained below; for each collared zigzag image, for the full circle of collarette zigzag versus its lower semicircle. The result shows that the best accuracy is found with the samples when using the lower semicircle of the collarette zigzag. Based on the results, our new idea that uses only the lower semicircle of the collarette achieved the highest recognition accuracy compared with the old methods that use the whole collarette region, $[2,16,18-23,25,51,58]$.

In this study case, we have done several training by increasing the number of classes: $(10,20,30,40)$ and keeping the number of samples always fixed, we notice that the accuracy decreases, on the other hand, if we increase the number of samples per class (something impossible with the CASIA database) the learning by CNN will improve greatly and the accuracy too.

Table 2. Accuracy; collarette full circle and collarette lower semicircle.

\begin{tabular}{ccc|}
\hline $\begin{array}{c}\text { Collarette: } \\
\text { Full circle }\end{array}$ & $\begin{array}{c}\text { Collarette:lower semi-circle } \\
\text { (our proposition) }\end{array}$ \\
\hline 100 & $93.33 \%$ & $100 \%$ \\
\hline 200 & $91.67 \%$ & $100 \%$ \\
\hline 300 & $88.89 \%$ & $100 \%$ \\
\hline 400 & $88.33 \%$ & $94.17 \%$ \\
\hline
\end{tabular}

Table 3. Performance parameters

\begin{tabular}{|ccccc}
\multicolumn{5}{c}{ Collarette: Full circle } \\
Sensitivity & Specificity & Precision & Recall & Fscore \\
\hline 0.91 & 0.997 & 0.88 & 0.91 & 0.87 \\
\hline & Collarette: lower semi-circle (our proposition) & \\
\hline Rensitivity & Specificity & Precision & Recall & Fscore \\
\hline 0.94 & 0.998 & 0.95 & 0.94 & 0.93 \\
\hline
\end{tabular}




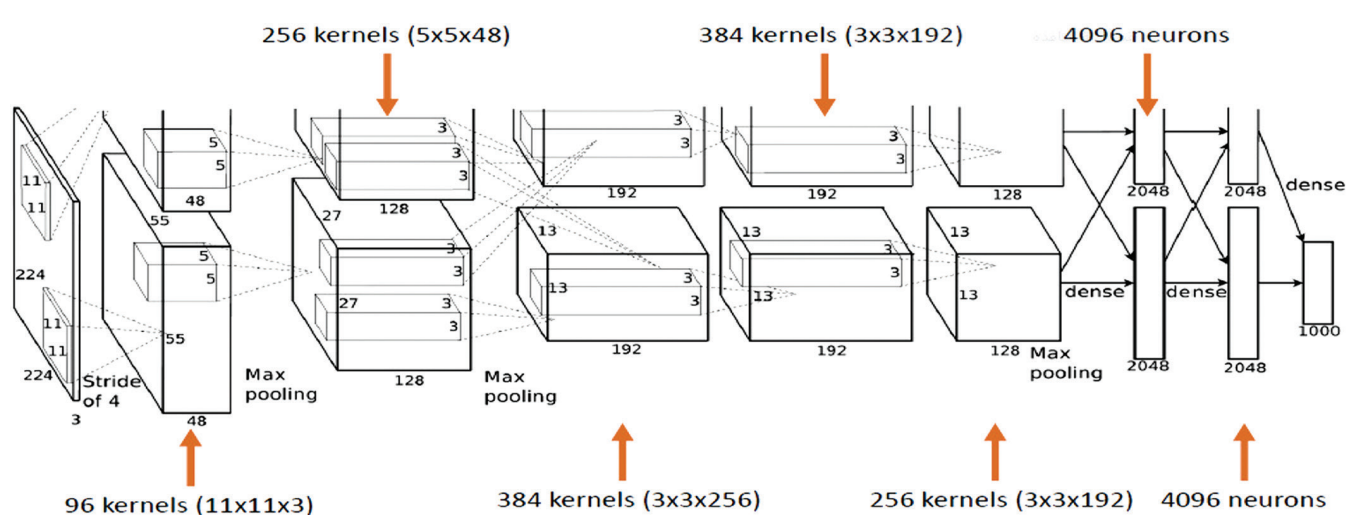

Fig. 9. Detailed architecture of Alex-Net [17]

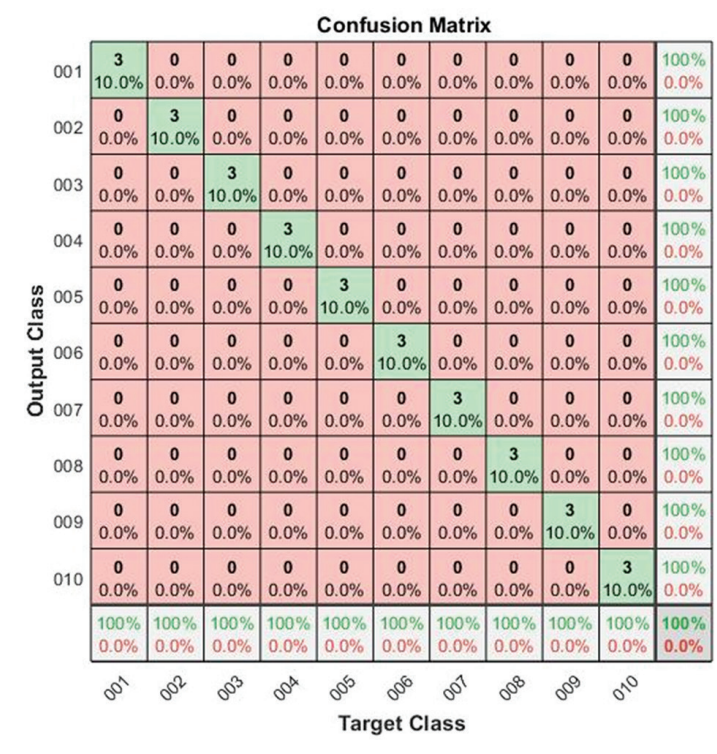

Fig. 10. Confusion matrix for first test (Collarette: lower semi-circle)

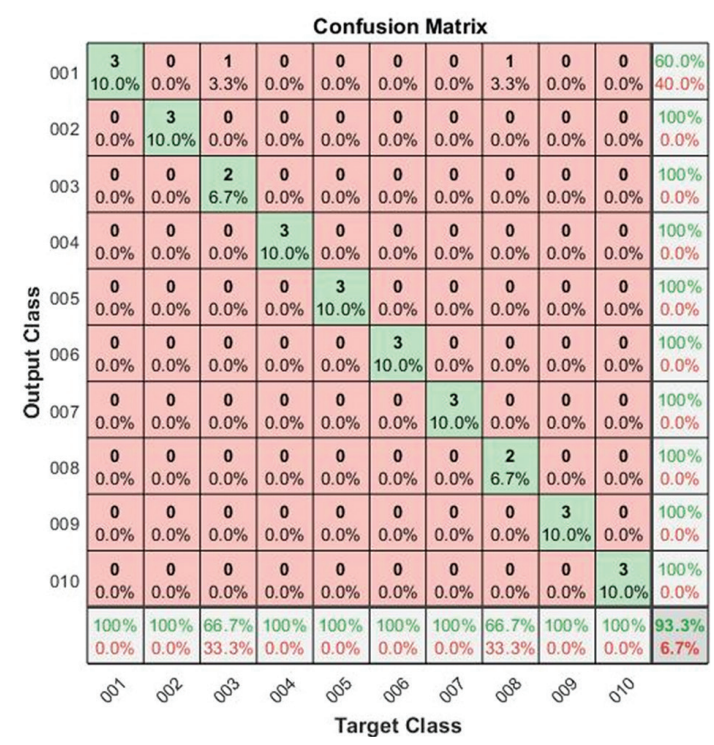

Fig. 11. Confusion matrix for second test (Collarette: Full circle)

In addition to the accuracy, the performance analysis of the two experiments (Collarette: Full circle/ Collarette: lower semi-circle) was based on the most used evalu- ation measures for statistical tests, such as (sensitivity, specificity, precision, recall, fscore ) [59] in order to further verify the performance of our CNN/SVM classifier, for those we performed the first test with 100 images (10 classes) -as an example- and compute the confusion matrix to display the classification results of our tests. By calculating the statistical parameters, the performance of our system is evaluated and presented in Table 3. After the analysis, we can easily see the superiority of all parameters in the case of the lower semi-circle of Collarette and also in the confusion matrix, see Fig. 10 and Fig. 11.

\section{CONCLUSION}

In this paper, we presented an iris recognition method for person identification based on CNN and the zigzag collarette region. The impact of the choice of the CNN characterization on the lower semicircle of the collarette region allowed us at the same time to target the least noisy area of the collarette with an optimal feature vector. We used an Alex-Net model pretrained on over a million images as a feature extractor, and a multi-class SVM for classification. Alex-Net with the SVM classifier achieved good accuracy with a fairly short learning time, on the order of seconds to minutes. The iris detection rate using the full semicircle of collarette zigzag was compared with the detection rate using only its lower semicircle. Images from the CASIAIris-Lamp V4 database were used to evaluate our approach. Version 4 of the CASIA database has been applied for the first time to study the contribution of the collarette zigzag area with the CNN for iris recognition.

The choice of lower semicircle of collarette had a very effective contribution on the accuracy as mentioned in Table 2, we notice that the accuraccy using lower semicircle of collarette is always higher than that of the full of circle collarette. The classification of the test set is represented by a confusion matrix. It shows the performance of a classifier on a test data set. If a class is mislabeled as the other class among several classes, we can easily identify it from a confusion matrix, other statistical parameters have been prospected; the results confirm our choice (low collarette/CNN) We have chosen the SVM algorithm because it can be adapted to classification problems involving more than 2 classes. In contract to a neural net- 
work which requires a lot of work to determine the right structure and parameters to use, SVM perform well even without any preparation. CNN facilitates the task of extracting the characteristics and making it easier to learn the new task assigned and extract more features from the image, allowing an SVM classifier to be better informed and achieve good accuracy. The crucial advantage of our combined approach (CNN/SVM) is that we can extract enough features (4096 features from the FC7 layer) from each image by representing the detail of each image from a pre-trained Alex-Net CNN model, and take advantage of the SVM to classify the features, saving time.

For future works, we plan to use data augmentation to increase the data artificially by learning a good amount of data. It is also interesting to train the data on other pre-trained models and finally use other databases.

\section{ACKNOWLEDGMENTS}

The authors would like to thank the DGRSDT (General Directorate of Scientific Research and Technological Development) - MESRS (Ministry of Higher Education and Scientific Research), ALGERIA, for the financial support of LERICA Laboratory( Laboratory for Study and Research in Instrumentation and Communication Annaba).

\section{REFERENCES}

[1] H. K. Rana, S. Azam, R. Akhtar, J. M. W, Quinn, M. A. Moni, "A fast iris recognition system through optimum feature extraction", PeerJ Computer Science, 2019

[2] A. Joshi, A. Gangwar, Z. Saquib, "Collarette Region Recognition based on Wavelets and Direct Linear Discriminant Analysis", International Journal of Computer Applications, Vol. 40, No. 9, 2012, pp.0975-8887.

[3] R. A. Ali, A. A. Maryoosh, D. N. George, W. R. Humood, "Iris images encryption based on QR code and chaotic map", TELKOMNIKA Telecommunication, Computing, Electronics and Control, 2020, Vol. 18 , No. 1, pp. 289-300.

[4] D. F. Santos, H. E. Espitia, "Detection of uveal melanoma using fuzzy and neural networks classifiers", TELKOMNIKA Telecommunication, Computing, Electronics and Control, 2020, Vol. 18, No. 4, pp. 2213-2223.

[5] P. S. Sanjekar, J. B. Patil, "Multimodal biometrics with serial, parallel and hierarchical mode at decision level fusion", Indonesian Journal of Electrical Engineering and Computer Science, Vol. 16, No. 3, 2019, pp. 1303-1310.
[6] H. Ohmaid, S. Eddarouich, A. Bourouhou, M. Timouyas, "Iris segmentation using a new unsupervised neural approach", IAES International Journal of Artificial Intelligence, Vol. 9, No. 1, 2020, pp. 5864.

[7] A. S. Akinfende, A. L. Imoize, O. S. Ajose, "Investigation of iris segmentation techniques using active contours for non-cooperative iris recognition", Indonesian Journal of Electrical Engineering and Computer Science, Vol. 19, No. 3, 2020, pp. 12751286.

[8] N. Bounegta, A. Basso, M. Beladgham, " Eyelids, eyelashes detection algorithm and hough transform method for noise removal in iris recognition", Indonesian Journal of Electrical Engineering and Computer Science, Vol. 18, No. 2,2020, pp. 731-735.

[9] R. F. Soliman, M. Amin, F. E. Abd El-Samie, "Cancelable Iris recognition system based on comb filter", Multimedia Tools and Applications, Vol. 79, 2019, pp. 2521-2541.

[10] S. Adamović, V. Miškovi, N. Maček, M. Milosavljević, M. Šarac, M. Saračević, M. Gnjatović, "An efficient novel approach for iris recognition based on stylometric features and machine learning techniques", Generation Computer Systems, Vol. 107, 2020, pp. 144-157.

[11] Y. Jusman, S. C. Ng, K. Hasikin, "Performances of proposed normalization algo-rithm for iris recognition", International Journal of Advances in Intelligent Informatics, Vol. 6, No. 2, 2020, pp. 161-172.

[12] N. Ahmadi, G. Akbarizadeh, "Iris tissue recognition based on GLDM feature extraction and hybrid MLPNN-ICA classifier", Neural Computing and Applications, Vol. 32, 2018, pp. 2267-2281.

[13] M. S. Chetali, R. V. Babar," Design of Iris Recognition Biometric System", International Journal of Research in Engineering, Science and Management, Vol. 2, No. 6, 2019, pp. 445-448

[14] A. Kour, B. Gupta, "Secure Vault System using Iris Biometrics and PIC Microcontroller", International Research Journal of Engineering and Technology, Vol. 6, No. 3, 2019.

[15] X. F. He, PF. Shi, "An Efficient Iris Segmen-tation Method for Recognition", International Conference on Pattern Recognition and Image Analysis, LNCS 3687, 2005, pp. 120-126. 
[16] H. Sung, J. Lim, J. Park, L. Yillbyung, "Iris Recognition Using Collarette Boundary Localization", Proceedings of the 17th International Conference on Pattern Recognition, Cambridge, UK, 26-26 August 2004.

[17] M. G. Alaslani, L. A. Elrefaei, "Convolutional neural network based feature extraction for iris recognition", International Journal of Computer Science \& Information Technology, Vol. 10, No 2, 2018, p. 10206.

[18] R. H. Jeng, S. W. Chen, L. Hsieh, "Does Collarette of Iris Work for Recognizing Persons?", Proceedings of the 7th International Multi-Conference on Computing in the Global Information Technology, 2012.

[19] H. Rai, A. Yadav, "Iris recognition using combined support vector machine and Hamming distance approach", Expert Systems with Applications, Vol. 41, 2014, pp.588-593.

[20] P. Punyani, A. Kumar, R. Gupta, "An optimized Iris Recognition System using MOGA followed by Combined Classifiers", International Journal of Research in Advent Technology, Vol. 4, No. 3, 2016.

[21] M. W. Frazier, "An Introduction to Wavelets through Linear algebra", Springer, 1999.

[22] H. Yu, J. Yang, "A Direct LDA Algorithm for High-Dimensional Data with Application to Face Recognition", Pattern Recognition, Vol. 34, 2001, pp. $2067-$ 2070.

[23] M. R. Faundra, D. R. Sulistyaningrum, "Iris Segmentation and Normalization Algorithm Based on Zigzag Collarette", Journal of Physics Conference Series, Vol. 795, 2017.

[24] K. Roy, P. Bhattacharya, "An Iris Recognition Method Based On Zigzag Collarette Area and Asymmetrical Support Vector Machines", Proceedings of the IEEE International Conference on Systems, Man, Cybernetics, Taipei, Taiwan, 8-11 October 2006.

[25] S. S. Jauro, R. Yadav, " An efficient interior and exterior boundary detection- based two level iris segmentation", International Journal of Information and Computer Security, Vol. 13, No.1, 2020.

[26] S. Minaee, A. Abdolrashidiy, Y. Wang, "An experimental study of deep convolutional features for iris recognition", Proceedings of the Signal Processing in Medicine and Biology Symposium, Philadelphia, PA, USA, 3 December 2016, pp. 1-6.
[27] S. Minaee, A. Abdolrashidi, Y. Wang, "Iris recognition using scattering transform and textural features", Proceedings of the Signal Processing and Signal Processing Education Workshop, Salt Lake City, UT, USA, 9-12 August 2015, pp. 37-42.

[28] S. Minaee, A, Abdolrashidi, Y. Wang, "Face Recognition Using Scattering Convolutional Network", arXiv preprint, 2016 arXiv:1608. 00059.

[29] H. Li, Z. Sun and T. Tan, "Robust iris segmen-tation based on learned boundary detectors", In Proceedings of the International Conference on Biometrics, New Delhi, India, 29 March-1 April 2012, pp. 317322.

[30] H. Vokhidov, H. G. Hong, J. K. Kang, T. M. Hoang, K. R. Park, "Recognition of damaged arrow-road markings by visible light camera sensor based on convolutional neural network", Sensors, Vol. 16, 2016, p. 2160.

[31] D, T. Nguyen, K, W. Kim, H, G. Hong, J, H. Koo, M, C. Kim, K, R. Park, "Gender recognition from humanbody images using visible-light and thermal camera videos based on a convolutional neural network for image feature extraction", Sensors, Vol. 17, 2017, p. 637.

[32] J. H. Kim, H. G. Hong, K.R. Park, "Convolutional neural network-based human detection in nighttime images using visible light camera sensors", Sensors, Vol. 17, 2017, pp. 1-26.

[33] B. Barros, P. Lacerda, C. Albuquerque, A. Conci, "Pulmonary COVID-19: Learning Spatiotemporal Features Combining CNN and LSTM Networks for Lung Ultrasound Video Classification", Sensors, Vol. 21, No. 16, 2021, p. 5486.

[34] S. Pereira, A. Pinto, V. Alves, CA. Silva, «Brain tumor segmentation using convolutional neural networks in MRI images", IEEE Transactions on Medical Imaging, Vol. 35, 2016, pp. 1240-1251.

[35] M. Arsalan, H. G. Hong, R. A. Naqvi, M. B. Lee, M. C. Kim, D. S. Kim, C, S. Kim, K, R. Park, "Deep Learning-Based Iris Segmentation for Iris Recognition in Visible Light Environment", Symmetry, Vol. 9, 2017, p. 263.

[36] N. Liu, M. Zhang, H. Li, Z. Sun, T. Tan, "Deeplris: Learning pairwise filter bank for heterogeneous iris verification", Pattern Recognition Letters, Vol. 82, 2016, pp. 154-161. 
[37] A. Gangwar, A. Joshi, “DeeplrisNet: Deep iris representation with applications in iris recognition and cross-sensor iris recognition", Proceedings of the IEEE International Conference on Image Processing, Phoenix, AZ, USA, 25-28 September 2016, pp. 2301-2305.

[38] M. Pathak, N. Srinivasu, V. Bairagi, “Effective segmentation of sclera, iris and pupil in noisy eye images", TELKOMNIKA Telecommunication, Computing, Electronics and Control, Vol. 17, No. 5, 2019, pp. 2346-2354.

[39] M. Trokielewicz, A. Czajka, P. Maciejewicz, "Postmortem Iris Recognition with Deep-Learningbased Image Segmentation", Image and Vision Computing, Vol. 94, 2019.

[40] J. Rahman, M. Noridayu, B. Manshor and Li. S. Affendey, "Iris Recognition Development Techniques: A Comprehensive Review", Complexity, $2021,6641247$.

[41] A. S. Al-Waisy, R. Qahwaji, S. Ipson, S. Al-Fahdawi, T. A. Nagem, "A multi-biometric iris recognition system based on a deep learning approach", Pattern Analysis and Applications, Vol. 27, 2018, pp. 783-802.

[42] K. Nguyen, C. Fookes, A. Ross, S. Sridharan, "Iris Recognition with Off-the-Shelf CNN Features: A Deep Learning Perspective", IEEE Access, Vol. 6, 2017, pp. 18848-18855.

[43] CASIA iris database, http://www.idealtest.org/ findTotalDbByMode.do?mode=Iris, (accessed: 2012).

[44] UBIRIS iris database, http://iris.di.ubi.pt/, (accessed: 2012).

[45] IITD iris database, http://www.iitd.ac.in/, (accessed: 2012).

[46] MMU iris databases, http://pesona.mmu.edu. my/ ccteo/, (accessed: 2012).

[47] J, G. Daugman, “High confidence visual recognition of persons by a test of statistical independence", IEEE Transactions on Pattern Analysis and Machine Intelligence, Vol. 15, 1993, pp. 1148-1161.

[48] K. Roy, P. Bhattacharya, "Collarette Area Localization and Asymmetrical Support Vector Machines for Efficient Iris Recognition", Proceedings of the 14th International Conference on Image Analysis and Processing, Modena, Italy, 10-14 September 2007.
[49] W. Sankowski, K. Grabowski, M. Napieralska, M. Zubert, A. Napieralski, ", Reliable algorithm for iris segmentation in eye image", Image and Vision Computing, Vol. 28, 2010, pp. 231-237.

[50] P. D. Almeida, "A knowledge-based approach to the iris segmentation problem", Image and Vision Computing, Vol. 28, 2010, pp. 238-245.

[51] Z. He, T. Tan, Z. Sun, X. Qiu, "Toward accurate and fast iris segmentation for iris biometrics", IEEE Transactions on Pattern Analysis and Machine Intelligence, Vol. 31, 2009, pp. 1670-1684.

[52] F. Jan, I. Usman, S. Agha, "Iris localization in frontal eye images for less constrained iris recognition systems", Digital Signal Processing, Vol. 22, 2012, pp. 971-986.

[53] N. Singh, D. Gandhi, K. P. Singh, "Iris recognition system using a canny edge detection and a circular Hough transform", International Journal of Advances in Engineering \& Technology, Vol. 1, No. 2, 2011, pp. $221-228$.

[54] S. Sangwan, R. Rani, "A Review on: Iris Recognition", International Journal of Computer Science and Information Technologies, Vol. 6, 2015, pp. 3871-3873.

[55] A. S. Al-Waisy, R. Qahwaji, S. Ipson, S. Al-Fahdawi, "A fast and accurate iris localization technique for healthcare security system", Proceedings of the IEEE International Conference on Computer and Information Technology; Ubiquitous Computing and Communications; Dependable, Autonomic and Secure Computing; Pervasive Intelligence and Computing, Liverpool, UK, 26-28 October 2015, pp 1028-1034

[56] S. Minaee, Y. Wang, "Palmprint Recognition Using Deep Scattering Convolutional Network", arXiv preprint, 2016 arXiv:1603. 09027.

[57] A. Krizhevsky, I. Sutskever, G, E. Hinton, "Imagenet classification with deep convolutional neural networks", Communications of the ACM, Vol. 60, No. 6, 2017, pp. 84-90.

[58] D. S. Jeong, J. W. Hwang, B. J. Kang, K. R. Park, C, S. Won, D, K. Park, J. Kim, "A new iris segmentation method for non-ideal iris images", Image and Vision Computing, Vol. 28, 2010, pp. 254-260.

[59] S. Almabdy, L. Elrefaei, “Deep convolutional neural network-based approaches for face recognition", Applied Sciences, 2019, Vol. 9, No. 20, p. 4397. 